\title{
Hepatic Myelopathy with Painful Tonic Spasm: A Case Report
}

\author{
Huanquan Liao ${ }^{*}$, Zhichao Yan ${ }^{2 *}$, Guidian $\mathrm{Li}^{3 *}$, Hua Hong1\# \\ ${ }^{1}$ Department of Neurology, First Affiliated Hospital, Sun Yat-Sen University, Guangzhou, China \\ ${ }^{2}$ State Key Laboratory of Ophthalmology, Zhongshan Ophthalmic Center, Sun Yat-sen University, \\ Guangzhou, China \\ ${ }^{3}$ Department of Radiology, East Hospital, First Affiliated Hospital, Sun Yat-Sen University, Guangzhou, China \\ Email: "
}

Received 4 October 2015; accepted 20 October 2015; published 27 October 2015

Copyright (C) 2015 by authors and OALib.

This work is licensed under the Creative Commons Attribution International License (CC BY).

http://creativecommons.org/licenses/by/4.0/

(c) $\underset{\mathrm{EY}}{\mathrm{EY}}$ Open Access

\begin{abstract}
Hepatic myelopathy (HM) is a rare neurological complication of chronic liver disease, causing progressive spastic paraplegia or quadriplegia in the extremities with minimal sensory or sphincter impairment. In this article, the authors present one additional case with HM, with an exceptional clinical picture of painful tonic spasms within both thighs as the main complaint. A reduction in pain coinciding with a reduction in spasticity from treatments such as baclofen suggests a causal relationship between the two. We stress the importance of early recognizing this symptom before the spinal cord damage becomes irreversible.
\end{abstract}

\section{Keywords}

Hepatic Myelopathy, Painful Tonic Spasms, Upper Motor Neuron Syndrome

Subject Areas: Neurology, Pathology

\section{Introduction}

Patients with chronic liver disease (CLD) frequently experience neurologic sequelae. The most common and widely recognized is the reversible syndrome of hepatic encephalopathy (HE). There are also comparatively rare and largely irreversible conditions such as acquired hepatocerebral degeneration [1] and hepatic myelopathy (HM) [2]. The typical scenario of HM is the progressive spasticity and paraplegia (sometimes quadriplegia) in the extremities due to a symmetric myelin loss in the lateral corticospinal tracts of the spinal cord. Upper motor neuron syndrome (UMNS), which is attributed to the similar damage to the upper motor neurons or the des-

\footnotetext{
*These authors contributed equally.

${ }^{\#}$ Corresponding author.
} 
cending corticospinal system, results in neurological motor overactivity including spasticity [3]. Pain, as a component of UMNS, sometimes replaced by other disagreeable sensations specified as cramp, tingling, numbness, or discomfort, is a frequently cited indication for the treatment of spasticity such as multiple sclerosis (MS) [4], spinal cord injury [5], traumatic brain injury and stroke [6]. Herein, the authors present an additional case of HM, apart from quadriparesis, with an exceptional clinical picture of painful tonic spasm accompanied by a disagreeable numbness sensation within both thighs.

\section{Case Report}

A 45-year-old man was admitted on March 6, 2014, because of a tingling sensation within both thighs which had proceeded over the previous 3 months. The attacks were brief, tingling or lance like, lasting less than 2 minutes, and might occur as frequently as several times per hour, prominent during nighttime. In addition, he felt weak and disagreeably numb in the lower extremities, though ambulatory in his daily life. He was once treated as an in-patient in several other hospitals with suspected diagnoses of "lateral femoral cutaneous neuritis", "myofascial pain syndrome" respectively. Nevertheless the outcome seemed to be unrewarding. Gradually his nocturnal leg cramps became more serious, and his walking became unsteady, tripping over objects. On admission he could still walk without help. There was no sphincter or erectile dysfunction. He was diagnosed as chronic hepatitis B, and found to have elevated plasma aminotransferase when he was treated in another hospital 3 months ago. There was neither history of liver cirrhosis nor recurrent prior episodes of hepatic encephalopathy nor recurrent jaundice or hematemesis. He had neither personal history of alcohol consumption nor family history of liver and neurological illnesses. On examination, the patient was alert, cooperative and orientated. He had no speech disturbance. There was no palmar erythema, spider-agioma, nor peripheral edema. The abdomen was flat without small venous collaterals. The spleen, liver and kidney were not palpable. There were no Kayser-Fleischer corneal rings. On neurological examination, cranial nerve examinations were normal. Spastic quadriparesis was observed. Muscle tone was increased. Tendon reflexes in all four extremities were brisk with respective Rossolimo sign and Babinski sign positive. Muscle strength in the proximal limbs was decreased (MRC grade 4). There was no clonus. In addition to spastic quadriparesis, the patient had pin-prick and touch sensory deficit within the lower extremities though that to the vibratory stimulation was still preserved. There was neither atrophy nor fasciculation. There was no evidence of extrapyramidal dysfunction. Hemogram was normal. Coagulation status showed no impairment. Liver function tests revealed raised alanine aminotransferase and aspartate aminotransferase. Serum bilirubin, ammonia and albumin level were normal. Serum Vitamin B12 and foliate values were within normal ranges. Serum antibodies to human T cell lymphotrophic virus (HTLV-1) and Hepatitis C virus were absent. HBs antibody was negative, and HBsAg, HBc antibody, HBe antibody were positive. Copper values of serum and urine were normal. Cerebrospinal fluid examination (pressure, Pandy, protein, glucose levels, cell counts, and IgG index analysis) revealed no abnormality. Figure 1 showed that Abdomen CT exhibited features of liver cirrhosis. EEG was normal. Figure 2 showed that Cranial and whole spinal cord MRI showed no abnormalities. Nerve conduction and electromyography was normal. The emission computerized tomographic bone imaging and color Doppler study of the blood vessels in the lower extremities showed no abnormality. After several weeks of liver protection, together with oral baclofen, the patient's pain got relieved, but the neurological examination remained unchanged. He was discharged one month later. On the basis

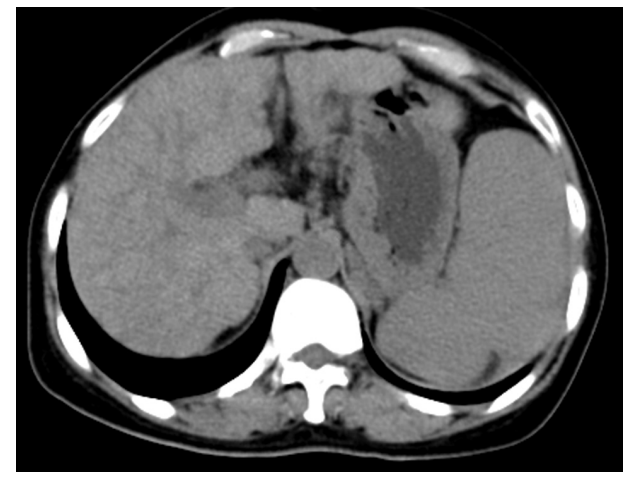

Figure 1. Abdomen CT exhibited features of liver cirrhosis. 


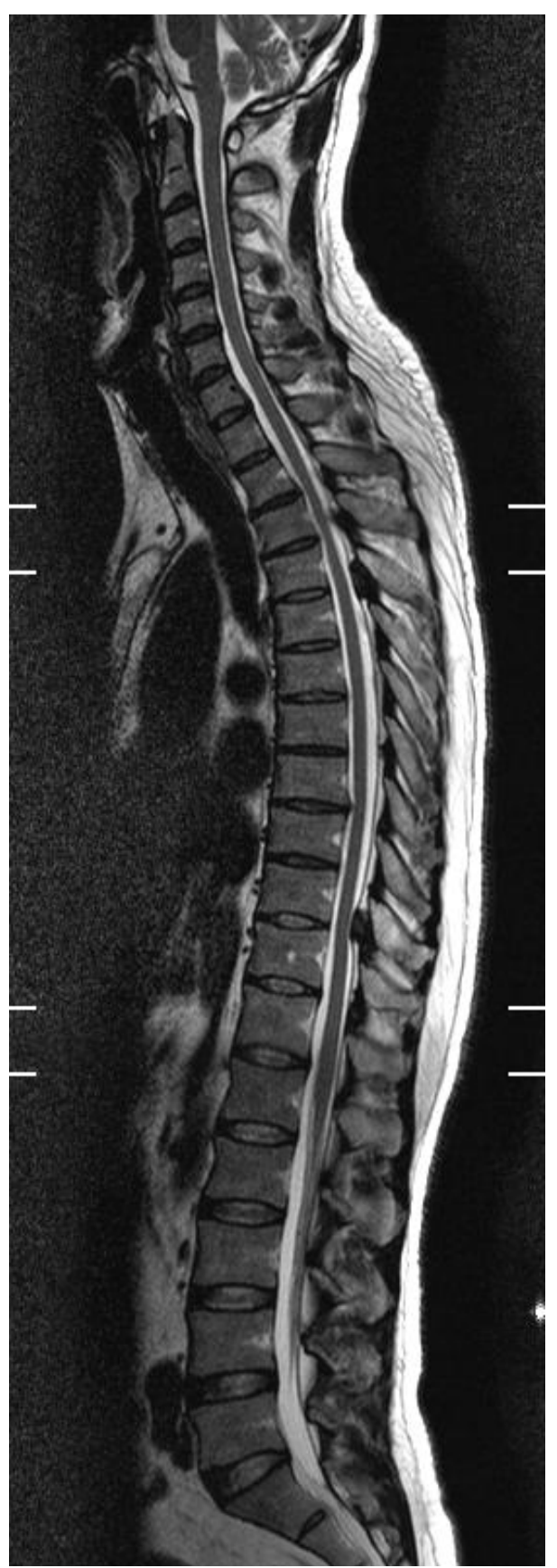

Figure 2. Cranial and whole spinal cord MRI showed no abnormalities.

of the above-mentioned information, taking the current liver disease into consideration and by exclusion of other possible causes, we located the lesion responsible in the spinal cord, and established the diagnosis of HM, attributing the pain to the muscle spasticity. It seemed that baclofen, a derivative of gamma-aminobutyric acid primarily used to treat spasticity, produced a marked effect.

\section{Discussion}

HM is a rare neurological complication of CLD, causing progressive spastic paraplegia or quadriplegia in the extremities with minimal sensory or sphincter impairment [2]. There is no special diagnostic tool for HM and it has to be established presumptively on clinical grounds after exclusion of other possible causes of spastic para- 
lysis. Cranial MRI of our case showed no abnormalities, eliminating latent brain lesions. Negative spinal cord MRI supported HM in the differential diagnosis, because MRI was essential to rule out such etiologies involving infarction, myelitis or compression of the spinal cord (epidural cord compression or intrinsic cord tumor). The most common scenario of MS is sensory symptoms, optic neuritis, extensive transverse myelitis including motor or bladder involvement, brain stem symptoms, and Lhermitte's phenomenon [7]. Brain MRI is abnormal in the majority of patients with MS. Visual evoked potentials, oligoclonal bands, and spinal cord MRI are useful if the MRI brain is negative and in patients with a progressive presentation. Our case did not show any of the above manifestations or abnormalities. Amyotrophic lateral sclerosis (ALS) patients complain, most often, of progressive weakness in the distal limbs. However, weakness may manifest in any body segment (bulbar, cervical, thoracic, or lumbosacral). Nerve conduction and electromyography studies improve diagnostic sensitivity. However, the result of the present case is normal. Other entities which is needed to be excluded include hereditary spastic paraplegia, subacute combined degeneration, Wilson syndrome, and human T cell lymphoma virus 1 (HTLV-1) infection, but there was no family history of neurological disorders, serum Vitamin B12 and folate values were in normal ranges, copper values of serum and urine were normal, and serum antibody to human $\mathrm{T}$ cell lymphotrophicvirus (HTLV-1) was absent.

It was suggested that the features in our patient were plausible HM: spastic quadriparesis, which was progressive and permanent, directly presenting to us as an unusual initial form of presentation of liver cirrhosis. Although the HM patients with liver cirrhosis manifested symptoms usually in the end stage of liver disease with prior evidence of liver dysfunction, through reviewing the literature, we still found 15 reported cases without cirrhosis [8]-[13] and 2 cases similarly presenting with neurologic dysfunction as an unusual initial form of presentation of liver cirrhosis [14]. Cirrhosis often presents insidiously. Therefore, the physicians discover latent cirrhosis coincidentally during evaluation of some other unrelated disease in $20 \%$ of cirrhotic patients [15].

The present case differed from the previously reported HM in the presence of the symptom of remarkable pain attributed to the muscle spasticity which was relieved by baclofen. The damage to the upper motor neurons or the descending corticospinal system often results in neurological motor overactivity including spasticity [3]. Pain, sometimes replaced by other disagreeable sensations specified as cramp, tingling, numbness, or discomfort, is a frequently cited indication for the treatment of spasticity in the UMNS which is attributed to the damage of the similar lesions and frequently described in MS [4], spinal cord injury [5], traumatic brain injury and stroke [6]. A reduction in pain coinciding with a reduction in spasticity from treatments such as baclofen suggests a causal relationship between the two [16]. Since Leigh and Card [2] firstly recognized myelopathy in patients with liver failure in 1949 and Zieve [17] described the pathological findings in 1960, a consensus has been established that a symmetric myelin loss in the lateral corticospinal tracts of the spinal cord result in the progressive spasticity and paraplegia (sometimes quadriplegia) in the extremities. Increased muscle tone and hyperreflexia are the most common findings. Various plantar responses including flexor responses have been described [18] [19]. Painful tonic spasms, as an unusual form of manifestation, has been described at a glance incidentally but never emphasized in isolated literatures [20]-[24]. The remarkable pain of our patient is noteworthy because of its demonstrating that, HM, with damage to corticospinal tracts of the spinal cord as the main pathophysiological mechanism, can also manifest painful tonic spasm, one component of the UMNS. Although the concurrence of pain and spasticity is well-known, the pathophysiological mechanisms are not [16]. However, there are some plausible possibilities such as pain from intermittent muscle contraction, musculoskeletal consequences of abnormal postures (due to spasticity and spastic dystonia), or secondary tissue damage from abnormal postures [16].

Unlike HE, the conservative treatment of HM is usually considered inefficient. Liver transplantation should be considered as the first therapeutic option and performed before the spinal cord damage becomes irreversible [25] [26]. Pharmacologic agents that can be administered through different routes play an important part in treatment aimed at relieving spasticity, pain, and minimizing functional deficits caused by UMNS. Such options including baclofen [27], gabapentin [28], synthetic cannabinoid [29], and botulinum toxin A [30] and so on may be tried to improve mobility with decreased spasticity, although the effectiveness is not confirmed.

\section{Conclusion}

HM patients may present with an exceptional clinical picture of painful tonic spasms within both thighs as the main complaint. A reduction in pain coinciding with a reduction in spasticity from treatments such as baclofen 
suggests a causal relationship between the two. We stress the importance of early recognizing this symptom before the spinal cord damage becomes irreversible.

\section{Acknowledgements}

This study was supported in part by Guangdong Province Technological Grant 2007B031502003, Guangzhou City Technological Grant 2009ZL-E021, and National Natural Sciences Foundation of China Grant 30971028 (Hua Hong).

\section{References}

[1] Victor, M., Adams, R.D. and Cole, M. (1965) The Acquired (Non-Wilsonian) Type of Chronic Hepatocerebral Degeneration. Medicine (Baltimore), 44, 345-396. http://dx.doi.org/10.1097/00005792-196509000-00001

[2] Leigh, A.D. and Card, W.I. (1949) Hepato-Lenticular Degeneration: A Case Associated with Postero-Lateral Column Degeneration. Journal of Neuropathology \& Experimental Neurology, 8, 338-346. http://dx.doi.org/10.1097/00005072-194907000-00007

[3] Ivanhoe, C.B. and Reistetter, T.A. (2004) Spasticity: The Misunderstood Part of the Upper Motor Neuron Syndrome. American Journal of Physical Medicine \& Rehabilitation, 83, S3-S9. http://dx.doi.org/10.1097/01.PHM.0000141125.28611.3E

[4] Spissu, A., Cannas, A., Ferrigno, P., Pelaghi, A.E. and Spissu, M. (1999) Anatomic Correlates of Painful Tonic Spasms in Multiple Sclerosis. Movement Disorders, 14, 331-335. http://dx.doi.org/10.1002/1531-8257(199903)14:2<331::AID-MDS1020>3.0.CO;2-H

[5] Middleton, J.W., Siddall, P.J., Walker, S., Molloy, A.R. and Rutkowski, S.B. (1996) Intrathecal Clonidine and Baclofen in the Management of Spasticity and Neuropathic Pain Following Spinal Cord Injury: A Case Study. Archives of Physical Medicine and Rehabilitation, 77, 824-826. http://dx.doi.org/10.1016/S0003-9993(96)90264-6

[6] Kellett, M.W., Young, G.R. and Fletcher, N.A. (1997) Painful Tonic Spasms and Pure Motor Hemiparesis Due to Lacunar Pontine Infarct. Movement Disorders, 12, 1094-1096. http://dx.doi.org/10.1002/mds.870120646

[7] Palace, J. (2001) Making the Diagnosis of Multiple Sclerosis. Journal of Neurology, Neurosurgery \& Psychiatry, 71, i3-i8.

[8] Sage, J.I., Van Uitert, R.L. and Lepore, F.E. (1984) Alcoholic Myelopathy without Substantial Liver Disease. A Syndrome of Progressive Dorsal and Lateral Column Dysfunction. Archives of Neurology, 41, 999-1001. http://dx.doi.org/10.1001/archneur.1984.04050200109030

[9] Tsuchiya, K., Watabiki, S., Shiojiri, T., Matsumoto, A. and Tsukagoshi, H. (1993) Alcoholic Cerebellar Degeneration with Pyramidal Sign-In Relation to Alcoholic Myelopathy. No to Shinkei, 45, 169-175.

[10] Anand, B.A., Agarwala, S. and Nundy, S. (1992) Encephalomyelopathy Following Portocaval Shunt in Noncirrhotic Portal Fibrosis: A Case Report. Tropical Gastroenterology, 13, 152-154.

[11] Gauthier, G. and Wildi, E. (1975) Porto-Systemic Encephalo-Myelopathy. Revue Neurologique Société de Neurologie de Paris, 131, 319-338.

[12] Lewis, M.B., Macquillan, G., Bamford, J.M. and Howdle, P.D. (2000) Delayed Myelopathic Presentation of the Acquired Hepatocerebral Degeneration Syndrome. Neurology, 54, 1011. http://dx.doi.org/10.1212/wnl.54.4.1011

[13] Tazawa, K.I., Shimojima, Y., Okano, T., Yazaki, M., Takei, Y.I., Shimojo, H., et al. (2007) An Autopsy Case with Adult Onset Type II Citrullinemia Showing Myelopathy. Journal of the Neurological Sciences, 253, 77-80. http://dx.doi.org/10.1016/j.jns.2006.11.014

[14] Utku, U., Asil, T., Balci, K., Uzunca, I. and Celik, Y. (2005) Hepatic Myelopathy with Spastic Paraparesis. Clinical Neurology and Neurosurgery, 107, 514-516. http://dx.doi.org/10.1016/j.clineuro.2004.10.002

[15] Iwamura, K. and Inaba, R. (1983) Clinical Study on Latent Cirrhosis of the Liver. Tokai Journal of Experimental and Clinical Medicine, 8, 281-291.

[16] Sheean, D.G. (2009) Is Spasticity Painful? European Journal of Neurology, 16, 157-158. http://dx.doi.org/10.1111/j.1468-1331.2008.02371.x

[17] Zieve, L., Mendelson, D.F. and Goepfert, M. (1960) Shunt Encephalomyelopathy. II. Occurrence of Permanent Myelopathy. Annals of Internal Medicine, 53, 53-63. http://dx.doi.org/10.7326/0003-4819-53-1-53

[18] Liversed, L.A. and Rawson, M.D. (1966) Myelopathy in Hepatic Disease and Portosystemic Venous Anastomosis. The Lancet, 287, 277-279. http://dx.doi.org/10.1016/S0140-6736(66)90636-2

[19] Mendoza, G., Marti-Fabregas, J., Kulisevsky, J. and Escartin, A. (1994) Hepatic Myelopathy: A Rare Complication of 
Portacaval Shunt. European Neurology, 34, 209-212. http://dx.doi.org/10.1159/000117040

[20] Campellone, J.V., Lacomis, D., Giuliani, M.J. and Kroboth, F.J. (1996) Hepatic Myelopathy Case Report with Review of the Literature. Clinical Neurology and Neurosurgery, 98, 242-246. http://dx.doi.org/10.1016/0303-8467(96)00018-2

[21] Lebovics, E., Dematteo, R.E., Schaffner, F. and Gendelman, S. (1985) Portal-Systemic Myelopathy after Portacaval Shunt Surgery. Archives of Internal Medicine, 145, 1921-1922. http://dx.doi.org/10.1001/archinte.1985.00360100195034

[22] Counsell, C. and Warlow, C. (1996) Failure of Presumed Hepatic Myelopathy to Improve after Liver Transplantation. Journal of Neurology, Neurosurgery \& Psychiatry, 60, 590. http://dx.doi.org/10.1136/jnnp.60.5.590

[23] Panicker, J., Sinha, S., Taly, A.B., Ravishankar, S. and Arunodaya, G.R. (2006) Hepatic Myelopathy: A Rare Complication Following Extrahepatic Portal Vein Occlusion and Lienorenal Shunt. Neurology India, 54, 298-300. http://dx.doi.org/10.4103/0028-3886.27159

[24] Weissenborn, K., Tietge, U.J.F., Bokemeyer, M., Mohammadi, B., Bode, U., Manns, M.P. and Caselitz, M. (2003) Liver Transplantation Improves Hepatic Myelopathy: Evidence by Three Cases. Gastroenterology, 124, 346-351. http://dx.doi.org/10.1053/gast.2003.50062

[25] Troisi, R., Debruyne, J. and de Hemptinne, B. (1999) Improvement of Hepatic Myelopathy after Liver Transplantation. New England Journal of Medicine, 340, 151. http://dx.doi.org/10.1056/nejm199901143400216

[26] Qu, B., Liu, C., Guo, L., Yang, Y., Li, J.H., Yu, L. and Lv, Y. (2009) The Role of Liver Transplantation in the Treatment of Hepatic Myelopathy: Case Report with Review of the Literature. Transplantation Proceedings, 41, 1987-1989. http://dx.doi.org/10.1016/j.transproceed.2009.01.105

[27] Chou, R., Peterson, K. and Helfand, M. (2004) Comparative Efficacy and Safety of Skeletal Muscle Relaxants for Spasticity and Musculoskeletal Conditions: A Systematic Review. Journal of Pain and Symptom Management, 28, 140-175. http://dx.doi.org/10.1016/j.jpainsymman.2004.05.002

[28] Mueller, M.E., Gruenthal, M., Olson, W.L. and Olson, W.H. (1997) Gabapentin for Relief of Upper Motor Neuron Symptoms in Multiple Sclerosis. Archives of Physical Medicine and Rehabilitation, 78, 521-524. http://dx.doi.org/10.1016/S0003-9993(97)90168-4

[29] Wissel, J., Haydn, T., Muller, J., Brenneis, C., Berger, T., Poewe, W. and Schelosky, L.D. (2006) Low Dose Treatment with the Synthetic Cannabinoid Nabilone Significantly Reduces Spasticity-Related Pain: A Double-Blind PlaceboControlled Cross-Over Trial. Journal of Neurology, 253, 1337-1341. http://dx.doi.org/10.1007/s00415-006-0218-8

[30] Wissel, J., Muller, J., Dressnandt, J., Heinen, F., Naumann, M., Topka, H. and Poewe, W. (2000) Management of Spasticity Associated Pain with Botulinum Toxin A. Journal of Pain and Symptom Management, 20, 44-49. http://dx.doi.org/10.1016/S0885-3924(00)00146-9 